\title{
NUMERICAL BOUNDARY LAYERS FOR HYPERBOLIC SYSTEMS IN 1-D
}

\author{
Claire Chainais-Hillairet $^{1}$ and Emmanuel Grenier ${ }^{1}$
}

\begin{abstract}
The aim of this paper is to investigate the stability of boundary layers which appear in numerical solutions of hyperbolic systems of conservation laws in one space dimension on regular meshes. We prove stability under a size condition for Lax Friedrichs type schemes and inconditionnal stability in the scalar case. Examples of unstable boundary layers are also given.
\end{abstract}

Mathematics Subject Classification. 65M, 35L.

Received: February 2, 2000. Revised: October 24, 2000.

\section{INTRODUCTION}

\subsection{Presentation of the problem}

The aim of this paper is to describe the asymptotic behavior of numerical approximations of systems of conservation laws in one space dimension, of the form

$$
\partial_{t} u+\partial_{x} f(u)=0
$$

where $f: \mathbb{R}^{d} \mapsto \mathbb{R}^{d}$ and $u$ is a vector valued function, $u:(x, t) \in \mathbb{R}_{+} \times \mathbb{R}_{+} \rightarrow \mathbb{R}^{d}$. We assume that $f \in$ $C^{\infty}\left(\mathbb{R}^{d}, \mathbb{R}^{d}\right)$ is uniformly lipschitzian on $\mathbb{R}^{d}$ and that $f$ has uniformly bounded second derivatives. Equation (1) must be completed with an initial data

$$
u(x, 0)=u_{0}(x)
$$

for every $x \geq 0$, where $u_{0}$ is a given smooth function. We must also provide boundary conditions at $x=0$. There are different ways to do this, and we refer to [1] and [3].

Let us first recall the approach of Gisclon and Serre in [4,5]: we consider solutions of (1) as limits of solutions of the following parabolic equation

$$
\partial_{t} u^{\varepsilon}+\partial_{x} f\left(u^{\varepsilon}\right)-\varepsilon \partial_{x x} u^{\varepsilon}=0
$$

with Dirichlet boundary condition

$$
u^{\varepsilon}(0, t)=0 \text {. }
$$

Keywords and phrases. Boundary layers stability.

1 UMPA, ENS-Lyon, 46, allée d'Italie, 69364 Lyon Cedex 07, France. 
As $\varepsilon$ goes to 0 , solutions $u^{\varepsilon}$ of $(3,4)$ converge, under some particular conditions, to solutions of (1) with boundary conditions

$$
u(0, t) \in \mathcal{C}_{\mathrm{vis}}
$$

where $\mathcal{C}_{\text {vis }}$, introduced in [5] and [3] is the set of vectors $v \in \mathbb{R}^{d}$ such that there exists a solution $w$ of

$$
\partial_{x} f(v+w)=\partial_{x x} w
$$

on $x>0$ satisfying $w(0)=-v$ and $w \rightarrow 0$ as $x \rightarrow+\infty$. Near $0, \mathcal{C}_{\text {vis }}$ is a manifold, whose tangent space is spanned by the eigenvectors of $f^{\prime}(0)$ with negative corresponding eigenvalues. We refer to [4], [8] and [9] for more details.

In this paper instead of looking at solutions of (1) as limits of solutions of a parabolic system (namely as limits of solutions of $(3,4)$ ), we consider solutions of (1) as limits of numerical solutions obtained by some numerical schemes like monotonic schemes or Lax Friedrichs type schemes with homogeneous Dirichlet boundary condition. As in [6] and [8], we assume that the initial condition $u_{0}$ is compatible with this numerical boundary condition: it means

$$
u_{0}(0)=0
$$

This assumption will be discussed later (see Sect. 2.4).

We prove, under a smallness condition, the convergence of numerical solutions to solutions of (1) with boundary condition

$$
u(0, t) \in \mathcal{C}_{\text {num }}
$$

(see (16)) which is a numerical counterpart of (5), as long as this solution remains smooth. Therefore we do not investigate shocks, but rather the existence and stability of numerical boundary layers in small time. These layers turn out to be stable under a smallness condition, except in the scalar case where no condition is required. Hence this paper is a rigorous justification of the formal analysis developed in [9] and is a numerical counterpart to [8]. It also completes the analysis of [6].

In all the paper we will assume that the boundary is noncharacteristic, i.e. that 0 is never an eigenvalue of $f^{\prime}$. We also assume that the system is symmetrizable:

$\exists \mathcal{S} \in C^{\infty}\left(\mathbb{R}^{d}, \mathcal{M}_{d \times d}\right)$ such that $\forall u \in \mathbb{R}^{d}$,

$$
\mathcal{S}(u) \text { is symmetric nonnegative definite, } \mathcal{S}(u) \gg I \text {, and } \mathcal{S}(u) f^{\prime}(u) \text { is symmetric. }
$$

Moreover, we assume that $\mathcal{S}$ is uniformly bounded with respect to $u$ (similar proofs can be fulfilled if we only assume that $\mathcal{S}$ is locally bounded in $u$ ).

\subsection{Numerical schemes}

Let us now detail the numerical schemes. We want to compute an approximate solution of $u(x, t)$, solution of (1). Therefore, we consider an uniform mesh of $\mathbb{R}_{+}$, which is constituted of cells $\left.\left.M_{i}=\right](i-1) h, i h\right]$ of size $h$, $i \geq 1$. The center of the cell $M_{i}$ is denoted by $x_{i}=(i-1 / 2) h$.

Let $k$ be the time step and let set $t^{n}=n k$. In order to discretize the hyperbolic system (1), we use the modified Lax-Friedrichs scheme. Therefore, we introduce the numerical flux $F \in C^{\infty}\left(\mathbb{R}^{d} \times \mathbb{R}^{d}, \mathbb{R}^{d}\right)$ defined by:

$$
F(u, v)=\frac{f(u)+f(v)}{2}-\frac{\lambda}{2}(v-u)
$$

with $\lambda$ large enough $\left(\lambda>\sup _{u \in \mathbb{R}^{d}}\left|f^{\prime}(u)\right|\right)$. 
In the scalar case, we don't restrict the study to the Lax-Friedrichs scheme. We just make the following assumptions on the numerical flux

$\left\{\begin{array}{l}\text { (i) } F(u, u)=f(u) \\ \text { (ii) } F(u, v) \text { is nondecreasing with respect to } u \text { and nonincreasing with respect to } v \text {. }\end{array}\right.$

Hypothesis $(11 \mathrm{i}$ ) ensures the consistency of the scheme (it is clearly satisfied by Lax-Friedrichs fluxes), hypothesis (11 ii) its monotony under a CFL condition.

In both cases, the scheme is the following

$$
\begin{gathered}
\frac{h}{k}\left(u_{i}^{n+1}-u_{i}^{n}\right)+\left(F\left(u_{i}^{n}, u_{i+1}^{n}\right)-F\left(u_{i-1}^{n}, u_{i}^{n}\right)\right)=0, \quad i \geq 1, \\
u_{0}^{n}=0, \quad u_{i}^{0}=u_{0}\left(x_{i}\right)
\end{gathered}
$$

and the numerical solution is defined by

$$
\begin{cases}u_{\text {num }}(x, t)=u_{i}^{n} & \text { on } M_{i} \times\left[t^{n}, t^{n+1}[, \forall i \geq 1, \forall n \in \mathbb{N}\right. \\ u_{\text {num }}(0, t)=u_{0}^{n} & \text { on }\left[t^{n}, t^{n+1}[, \forall n \in \mathbb{N}\right.\end{cases}
$$

We assume that the space step and the time step are linked by a CFL condition:

$$
k \leq C h
$$

where $C$, small enough, depends on $F$ (see $(33))$.

The analysis of the paper can be extended to the case where (13) is replaced by

$$
u_{i}^{0}=\frac{1}{\left|M_{i}\right|} \int_{M_{i}} u_{0}(x) \mathrm{d} x
$$

\subsection{Main results}

Let us define $\mathcal{C}_{\text {num }}$ to be the set of vectors $u$ such that there exists a solution $v(i)$ to

$$
\left.\begin{array}{c}
F(u+v(i), u+v(i+1))=f(u) \\
v(0)=-u \\
v(+\infty)=0
\end{array}\right\}
$$

(see Sect. 2.1 for details). This space has already been introduced by Joseph and LeFloch in [9]. The hyperbolic system (1)-(2) with boundary condition $u(0, t) \in \mathcal{C}_{\text {num }}$ is well posed.

Theorem 1.1 (Lax-Friedrichs scheme). Let $u^{\text {int }, 0}$ be a smooth solution on a time interval $[0, T]$ to (1) with initial data $u_{0}$ with compact support satisfying the compatibility assumption (7) and boundary condition $u^{\text {int }, 0}(0,.) \in$ $\mathcal{C}_{\text {num }}\left(\right.$ see (16)). Let $u_{\text {num }}$ be the approximate solution defined by (12), (13), (10) and (14) under the CFL condition (15).

Then, there exists $C_{0}$ depending only on $F$ such that, if on $[0, T]$

$$
\left|u^{i n t, 0}(0, t)\right| \leq C_{0}
$$

then $u_{\text {num }}$ converges to $u^{i n t, 0}$ in $L^{\infty}\left([0, T], L^{2}\left(\mathbb{R}_{+}\right)\right)$and, on $[0, T]$,

$$
u_{i}^{n}-u^{i n t, 0}\left(x_{i}, t^{n}\right)-u^{b, 0}\left(i, t^{n}\right)=O(h)
$$


where $u^{b, 0}\left(., t^{n}\right)$ is a solution to (16) with $u=u^{i n t, 0}\left(0, t^{n}\right)$.

In fact the proof of this theorem says more since it gives a complete expansion of $u_{i}^{n}$ in $h$ and $k$. We will not precise the regularity of $u^{i n t, 0}$. Basically we need $H^{s}$ regularity with $s$ large. The restriction to compactly supported initial data is a nonessential simplification which avoid to take care of the behavior near $+\infty$.

In the scalar case $d=1$, following an idea of Goodman [7] and Liu [11] we can remove the smallness assumption (17) and for every scheme whose fluxes satisfy (11) we have:

Theorem 1.2 (1D scheme). Let $u^{\text {int,0 }}$ be a smooth solution on a time interval $[0, T]$ to (1) with initial data $u_{0}$ with compact support satisfying the compatibility assumption (7) and boundary condition $u^{\text {int }, 0}(0,.) \in \mathcal{C}_{\text {num }}$. Let $u_{\text {num }}$ be the approximate solution defined by (12), (13), (11) and (14) under the CFL condition (15).

Then on $[0, T], u_{\text {num }}(., t)$ converges to $u^{i n t, 0}(., t)$ in $L^{\infty}\left([0, T], L^{2}\left(\mathbb{R}_{+}\right)\right)$and, on $[0, T]$,

$$
u_{i}^{n}-u^{i n t, 0}\left(x_{i}, t^{n}\right)-u^{b, 0}\left(i, t^{n}\right)=O(h)
$$

where $u^{b, 0}\left(., t^{n}\right)$ is solution to (16) with $u=u^{i n t, 0}\left(0, t^{n}\right)$.

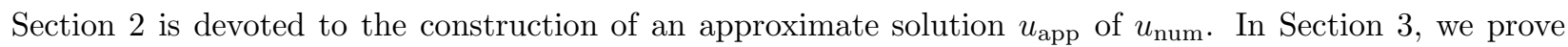
$L^{2}$ estimates between the numerical solution and the approximate one. Theorem 1.1 is then a consequence of this part. Theorem 1.2 is proved in Section 4. Section 5 is devoted to numerical counterexamples, that are cases of unstable boundary layers where the condition (17) is not satisfied, and Section 1.4 is a list of possible extensions.

\subsection{Extensions}

Similar techniques can be used in multidimensional space $x=\left(x_{1}, \ldots, x_{N}\right) \in \mathbb{R}^{N}$ with $x_{N} \geq 0$ in the particular case of regular meshes of rectangles. Theorem 1.1 can be extended to this case. We will not detail it here.

In one dimensional space $x \geq 0$, other boundary conditions can be treated, like inhomogeneous Dirichlet conditions

$$
u_{0}^{n}=g\left(t^{n}\right)
$$

where $g$ is some smooth given function, or Neumann conditions

$$
u_{0}^{n}=u_{1}^{n} .
$$

This latest case is even simpler since there is no boundary layer at leading order.

\section{Asymptotic Expansion}

Following [8], we want to construct some $u^{i n t, j}$ and $u^{b, j}$ such that

$$
u_{\mathrm{app}}\left(x_{i}, t^{n}\right)=\sum_{j=0}^{N} h^{j}\left(u^{i n t, j}\left(x_{i}, t^{n}\right)+u^{b, j}\left(i, t^{n}\right)\right)
$$

is a "good" approximation of $u_{i}^{n}$. The $u^{i n t, j}: \mathbb{R}_{+} \times \mathbb{R}_{+} \rightarrow \mathbb{R}^{d}$ are smooth functions and describe the numerical solution far away from the boundaries. The $u^{b, j}: \mathbb{N} \times \mathbb{R}_{+} \rightarrow \mathbb{R}^{d}$ are boundary layer profiles and therefore must be rapidly decreasing with respect to $i$.

In this section, we give a formal derivation of the equations satisfied by the $u^{i n t, j}$ and $u^{b, j}$. The fact that $u_{\text {app }}$ is really a good approximation of $u_{\text {num }}$ will be proved in Section 3 . The idea is the following: if $u_{\text {app }}\left(x_{i}, t^{n}\right)$ is close to $u_{\text {num }}\left(x_{i}, t^{n}\right)$ then it satisfies an equality similar to (12) but with some error terms. It means that, for all $i$ and $n, E_{i}^{n}$ defined by

$E_{i}^{n}=\frac{h}{k}\left(u_{\text {app }}\left(x_{i}, t^{n+1}\right)-u_{\text {app }}\left(x_{i}, t^{n}\right)\right)+\left(F\left(u_{\text {app }}\left(x_{i}, t^{n}\right), u_{\text {app }}\left(x_{i+1}, t^{n}\right)\right)-F\left(u_{\text {app }}\left(x_{i-1}, t^{n}\right), u_{\text {app }}\left(x_{i}, t^{n}\right)\right)\right)$ 
is controlled by $o\left(h^{\alpha(N)}\right)$ for some $\alpha(N)$. The approximate solution $u_{\text {app }}$ must also satisfy the initial and boundary conditions (13).

In the next subsections, we study the expansion of $E_{i}^{n}$ with respect to the powers of $h$ in each area of $\mathbb{R}_{+} \times \mathbb{R}_{+}$: in the boundary layer ( mall $i$ ) and outside the boundary layer (large $i$ ). If we want $E_{i}^{n}$ to be a $o\left(h^{\alpha(N)}\right)$, we have to set all the terms of the expansion of $E_{i}^{n}$ until $h^{\alpha(N)}$ equal to 0 : it formally yields the equations satisfied by $u^{i n t, j}$ and $u^{b, j}$. We also discuss the existence and uniqueness of the solutions to these equations.

\subsection{The first boundary layer profile: $u^{b, 0}$}

In order to get the equation on the first profile of the boundary layer, we study $E_{i}^{n}$ for $n$ large and $i$ small:

$$
\begin{aligned}
E_{i}^{n}=F\left(u^{i n t, 0}\left(x_{i}, t^{n}\right)+u^{b, 0}\left(i, t^{n}\right), u^{i n t, 0}\right. & \left.\left(x_{i+1}, t^{n}\right)+u^{b, 0}\left(i+1, t^{n}\right)\right) \\
& -F\left(u^{i n t, 0}\left(x_{i-1}, t^{n}\right)+u^{b, 0}\left(i-1, t^{n}\right), u^{i n t, 0}\left(x_{i}, t^{n}\right)+u^{b, 0}\left(i, t^{n}\right)\right)+O(h) .
\end{aligned}
$$

But, in the boundary layer, $u^{i n t, 0}\left(x_{i}, t^{n}\right)=u^{i n t, 0}\left(0, t^{n}\right)+O(h)$. Therefore,

$$
\begin{aligned}
E_{i}^{n}=F\left(u^{i n t, 0}\left(0, t^{n}\right)+u^{b, 0}\left(i, t^{n}\right), u^{i n t, 0}\left(0, t^{n}\right)\right. & \left.+u^{b, 0}\left(i+1, t^{n}\right)\right) \\
& -F\left(u^{i n t, 0}\left(0, t^{n}\right)+u^{b, 0}\left(i-1, t^{n}\right), u^{i n t, 0}\left(0, t^{n}\right)+u^{b, 0}\left(i, t^{n}\right)\right)+O(h)
\end{aligned}
$$

Setting the term of order 0 in $E_{i}^{n}$ equal to 0 gives the equation on $u^{b, 0}$. The following boundary condition is added: $u^{b, 0}\left(0, t^{n}\right)=-u^{i n t, 0}\left(0, t^{n}\right)$, which comes from the approximation of $u_{0}^{n}=0$, and $u^{b, 0}\left(i, t^{n}\right) \rightarrow 0$ when $i$ goes to $+\infty$, since $u^{b, 0}$ is rapidly decreasing with respect to $i$. Finally, $u^{b, 0}$ is solution to:

$$
\left.\begin{array}{c}
F\left(u^{i n t, 0}\left(0, t^{n}\right)+u^{b, 0}\left(i, t^{n}\right), u^{i n t, 0}\left(0, t^{n}\right)+u^{b, 0}\left(i+1, t^{n}\right)\right)=f\left(u^{i n t, 0}\left(0, t^{n}\right)\right) \\
u^{b, 0}\left(0, t^{n}\right)=-u^{i n t, 0}\left(0, t^{n}\right) \\
u^{b, 0}\left(+\infty, t^{n}\right)=0
\end{array}\right\}
$$

With such boundary conditions, there exist some solution $u^{b, 0}\left(., t^{n}\right)$ to the discrete dynamical system (20) only for some values of $u^{i n t, 0}\left(0, t^{n}\right)$, i.e. if and only if

$$
u^{i n t, 0}(0, .) \in \mathcal{C}_{\text {num }}
$$

which defines $\mathcal{C}_{\text {num. }}$. The space $\mathcal{C}_{\text {num }}$ is the numerical analog to $\mathcal{C}_{\text {vis }}$ (compare (6) with (16)). We refer to [9] for further properties of $\mathcal{C}_{\text {num. }}$. In particular $\mathcal{C}_{\text {vis }}$ and $\mathcal{C}_{\text {num }}$ have the same tangent space in 0 , which is spanned by the eigenvectors of $f^{\prime}(0)$ with negative corresponding eigenvalues.

\subsection{The first inner term: $u^{i n t, 0}$}

Note that the study of $u^{b, 0}$ gives the boundary condition on $u^{i n t, 0}: u^{i n t, 0}(0,.) \in \mathcal{C}_{\text {num }}$. The expansion of $E_{i}^{n}$ for large $i$ and $n$ leads to the equation satisfied by $u^{i n t, 0}$. Indeed, for such $i$ and $n$, all the boundary terms can be considered as equal to 0 . Using the smoothness of $u^{i n t, 0}, E_{i}^{n}$ rewrites:

$$
E_{i}^{n}=h \partial_{t} u^{i n t, 0}\left(x_{i}, t^{n}\right)+\left(F\left(u^{i n t, 0}\left(x_{i}, t^{n}\right), u^{i n t, 0}\left(x_{i+1}, t^{n}\right)\right)-F\left(u^{i n t, 0}\left(x_{i-1}, t^{n}\right), u^{i n t, 0}\left(x_{i}, t^{n}\right)\right)\right)+O\left(h^{2}\right)
$$

In this expression, we can add and subtract the quantity

$$
F\left(u^{i n t, 0}\left(x_{i+1}, t^{n}\right), u^{i n t, 0}\left(x_{i+1}, t^{n}\right)\right)-F\left(u^{i n t, 0}\left(x_{i}, t^{n}\right), u^{i n t, 0}\left(x_{i}, t^{n}\right)\right)
$$


On one hand, owing to the consistency of the numerical fluxes, we have:

$$
\begin{aligned}
F\left(u^{i n t, 0}\left(x_{i+1}, t^{n}\right), u^{i n t, 0}\left(x_{i+1}, t^{n}\right)\right)-F\left(u^{i n t, 0}\left(x_{i}, t^{n}\right), u^{i n t, 0}\left(x_{i}, t^{n}\right)\right) & = \\
f\left(u^{i n t, 0}\left(x_{i+1}, t^{n}\right)\right)-f\left(u^{i n t, 0}\left(x_{i}, t^{n}\right)\right) & =h f^{\prime}\left(u^{i n t, 0}\left(x_{i}, t^{n}\right)\right) . \partial_{x} u^{i n t, 0}\left(x_{i}, t^{n}\right)+O\left(h^{2}\right) .
\end{aligned}
$$

On the other hand, the regularity of $F$ and $u^{i n t, 0}$ and the regularity of the mesh imply

$$
\begin{aligned}
F\left(u^{i n t, 0}\left(x_{i}, t^{n}\right), u^{i n t, 0}\left(x_{i+1}, t^{n}\right)\right)- & F\left(u^{i n t, 0}\left(x_{i+1}, t^{n}\right), u^{i n t, 0}\left(x_{i+1}, t^{n}\right)\right) \\
& +F\left(u^{i n t, 0}\left(x_{i}, t^{n}\right), u^{i n t, 0}\left(x_{i}, t^{n}\right)\right)-F\left(u^{i n t, 0}\left(x_{i-1}, t^{n}\right), u^{i n t, 0}\left(x_{i}, t^{n}\right)\right)=O\left(h^{2}\right)
\end{aligned}
$$

Finally, we get:

$$
E_{i}^{n}=h\left(\partial_{t} u^{i n t, 0}\left(x_{i}, t^{n}\right)+\partial_{x}\left(f\left(u^{i n t, 0}\right)\right)\left(x_{i}, t^{n}\right)\right)+O\left(h^{2}\right) .
$$

We deduce that $u^{i n t, 0}$ must be solution to the hyperbolic conservation law (1) with initial and boundary conditions:

$$
\left.\begin{array}{c}
\partial_{t} u^{i n t, 0}+\partial_{x} f\left(u^{i n t, 0}\right)=0 \text { on } \mathbb{R}_{+} \times \mathbb{R}_{+}, \\
u^{i n t, 0}(., 0)=u_{0}(.) \text { on } \mathbb{R}_{+}, \\
u^{i n t, 0}(0, .) \in \mathcal{C}_{\text {num }} \text { on } \mathbb{R}_{+} .
\end{array}\right\}
$$

The definition of $\mathcal{C}_{\text {num }}$ ensures that the boundary condition on $u^{i n t, 0}\left(u^{i n t, 0}(0,.) \in \mathcal{C}_{\text {num }}\right)$ is maximal dissipative. Therefore, the problem (22) is well posed (locally in time) up to some compatibility conditions (as usual for hyperbolic systems in a half space). We refer to Rauch and Massey [12] or Li and Yu [10] for the expression of the compatibility conditions and the proof of this result.

\subsection{Equations on the next inner terms and profiles}

The boundary layer profiles: $u^{b, j}$

In order to obtain the equations on the next $u^{b, j}$, we go on with the expansion of $E_{i}^{n}$ with respect to the powers of $h$ for small $i$ and large $n$. For instance, the coefficient of $h$ in $E_{i}^{n}$ leads to the equation on $u^{b, 1}$ :

$$
\begin{aligned}
\partial_{1} F\left(u^{i n t, 0}\left(0, t^{n}\right)+\right. & \left.u^{b, 0}\left(i, t^{n}\right), u^{i n t, 0}\left(0, t^{n}\right)+u^{b, 0}\left(i+1, t^{n}\right)\right) u^{b, 1}\left(i, t^{n}\right) \\
& +\partial_{2} F\left(u^{i n t, 0}\left(0, t^{n}\right)+u^{b, 0}\left(i, t^{n}\right), u^{i n t, 0}\left(0, t^{n}\right)+u^{b, 0}\left(i+1, t^{n}\right)\right) u^{b, 1}\left(i+1, t^{n}\right) \\
& -\partial_{1} F\left(u^{i n t, 0}\left(0, t^{n}\right)+u^{b, 0}\left(i-1, t^{n}\right), u^{i n t, 0}\left(0, t^{n}\right)+u^{b, 0}\left(i, t^{n}\right)\right) u^{b, 1}\left(i-1, t^{n}\right) \\
& -\partial_{2} F\left(u^{i n t, 0}\left(0, t^{n}\right)+u^{b, 0}\left(i-1, t^{n}\right), u^{i n t, 0}\left(0, t^{n}\right)+u^{b, 0}\left(i, t^{n}\right)\right) u^{b, 1}\left(i, t^{n}\right)=S_{b, 1}\left(u^{b, 0}, u^{i n t, 0}(0, .)\right)
\end{aligned}
$$

with the following boundary conditions:

$$
u^{b, 1}\left(0, t^{n}\right)=-u^{i n t, 1}\left(0, t^{n}\right) \text { and } u^{b, 1}\left(+\infty, t^{n}\right)=0 .
$$

The source term $S_{b, 1}$ is a function of $u^{b, 0}$ and $u^{i n t, 0}(0,$.$) . It is not of wide interest to develop its expression here.$ We just want to note that it is rapidly decreasing with respect to $i$. The solution to this equation is in general not unique, and is the sum of a particular solution $u_{\text {par }}^{b, 1}\left(i, t^{n}\right)$ and a solution of the corresponding homogeneous 
equation (setting $S_{b, 1}$ to 0 ). The homogeneous equation, expressed in the variable $w_{i}$ is simply

$$
\begin{aligned}
\partial_{1} F\left(u^{i n t, 0}\left(0, t^{n}\right)+u^{b, 0}\right. & \left(i, t^{n}\right), u^{i n t, 0}\left(0, t^{n}\right) \\
+ & \partial_{2} F\left(u^{b, 0}\left(i+1, t^{n}\right)\right) w_{i} \\
& -\partial_{1} F\left(u^{i n t, 0}\left(0, t^{n}\right)+u^{b, 0}\left(i, t^{n}\right), u^{i n t, 0}\left(0, t^{n}\right)+u^{b, 0}\left(i-1, t^{n}\right), u^{i n t, 0}\left(0, t^{n}\right)+u^{b, 0}\left(i, t^{n}\right)\right) w_{i-1} \\
& -\partial_{2} F\left(u^{i n t, 0}\left(0, t^{n}\right)+u^{b, 0}\left(i-1, t^{n}\right), u^{i n t, 0}\left(0, t^{n}\right)+u^{b, 0}\left(i, t^{n}\right)\right) w_{i}=0
\end{aligned}
$$

Note that this equation is the linearized version of the equation on $u^{b, 0}$. Hence the set of solutions of the this homogeneous equation is of the dimension of $\mathcal{C}_{\text {num }}$, and the set of the values of $w_{0}$ is a subspace $\mathcal{P}_{u^{i n t, 0}\left(0, t^{n}\right)}$. Therefore

$$
u^{b, 1}\left(0, t^{n}\right)=-u^{i n t, 1}\left(0, t^{n}\right) \in u_{\mathrm{par}}^{b, 1}\left(0, t^{n}\right)+\mathcal{P}_{u^{i n t, 0}\left(0, t^{n}\right)}
$$

$u_{\text {par }}^{b, 1}\left(0, t^{n}\right)$ being given by the source term $S_{b, 1}$ which does not depend on $u^{b, 1}$ and $u^{i n t, 1}$.

Following the expansion on $E_{i}^{n}$, we will get the same kind of equation for all the $u^{b, k}$, with a source term $S_{b, k}$ still rapidly decreasing in $i$.

The inner terms: $u^{i n t, j}$

We formally obtain the equations on the $u^{i n t, j}$ by studying the different powers of $h$ in $E_{i}^{n}$ for large $i$ and $n$. For instance, to get the equation on $u^{i n t, 1}$, we are looking for the coefficient of $h^{2}$ in $E_{i}^{n}$.

For large $i$ and $n$, we have:

$$
\begin{aligned}
E_{i}^{n}= & h \partial_{t} u^{i n t, 0}\left(x_{i}, t^{n}\right)+\frac{h k}{2} \partial_{t t} u^{i n t, 0}\left(x_{i}, t^{n}\right)+h^{2} \partial_{t} u^{i n t, 1}\left(x_{i}, t^{n}\right) \\
& +\left(F\left(u^{i n t, 0}\left(x_{i}, t^{n}\right)+h u^{i n t, 1}\left(x_{i}, t^{n}\right), u^{i n t, 0}\left(x_{i+1}, t^{n}\right)+h u^{i n t, 1}\left(x_{i+1}, t^{n}\right)\right)\right. \\
& \left.-F\left(u^{i n t, 0}\left(x_{i-1}, t^{n}\right)+h u^{i n t, 1}\left(x_{i-1}, t^{n}\right), u^{i n t, 0}\left(x_{i}, t^{n}\right)+h u^{i n t, 1}\left(x_{i}, t^{n}\right)\right)\right)+O\left(h^{3}\right) .
\end{aligned}
$$

We add and substract $f\left(u^{i n t, 0}\left(x_{i+1}, t^{n}\right)\right)-f\left(u^{i n t, 0}\left(x_{i}, t^{n}\right)\right)$ in $E_{i}^{n}$. As $u^{i n t, 0}$ is solution to (1), it yields:

$$
E_{i}^{n}=h^{2} \partial_{t} u^{i n t, 1}\left(x_{i}, t^{n}\right)+\frac{h k}{2} \partial_{t t} u^{i n t, 0}\left(x_{i}, t^{n}\right)+\frac{h^{2}}{2} \partial_{x x}\left(f\left(u^{i n t, 0}\right)\right)\left(x_{i}, t^{n}\right)+\Delta_{F}+O\left(h^{3}\right)
$$

with

$$
\begin{aligned}
\Delta_{F}= & \left(F\left(u^{i n t, 0}\left(x_{i}, t^{n}\right)+h u^{i n t, 1}\left(x_{i}, t^{n}\right), u^{i n t, 0}\left(x_{i+1}, t^{n}\right)+h u^{i n t, 1}\left(x_{i+1}, t^{n}\right)\right)\right. \\
& \left.-F\left(u^{i n t, 0}\left(x_{i+1}, t^{n}\right), u^{i n t, 0}\left(x_{i+1}, t^{n}\right)\right)\right) \\
& -\left(F\left(u^{i n t, 0}\left(x_{i-1}, t^{n}\right)+h u^{i n t, 1}\left(x_{i-1}, t^{n}\right), u^{i n t, 0}\left(x_{i}, t^{n}\right)+h u^{i n t, 1}\left(x_{i}, t^{n}\right)\right)\right. \\
& \left.-F\left(u^{i n t, 0}\left(x_{i}, t^{n}\right), u^{i n t, 0}\left(x_{i}, t^{n}\right)\right)\right) .
\end{aligned}
$$

Using Taylor's formula, we can rewrite $\Delta_{F}$ as:

$$
\Delta_{F}=\Delta_{F}^{0}+\Delta_{F}^{1}
$$


with

$$
\begin{aligned}
\Delta_{F}^{0}=\left(F\left(u^{i n t, 0}\left(x_{i}, t^{n}\right), u^{i n t, 0}\left(x_{i+1}, t^{n}\right)\right)\right. & \left.-F\left(u^{i n t, 0}\left(x_{i+1}, t^{n}\right), u^{i n t, 0}\left(x_{i+1}, t^{n}\right)\right)\right) \\
& -\left(F\left(u^{i n t, 0}\left(x_{i-1}, t^{n}\right), u^{i n t, 0}\left(x_{i}, t^{n}\right)\right)-F\left(u^{i n t, 0}\left(x_{i}, t^{n}\right), u^{i n t, 0}\left(x_{i}, t^{n}\right)\right)\right)
\end{aligned}
$$

and

$$
\begin{aligned}
\Delta_{F}^{1}= & h \partial_{1} F\left(u^{i n t, 0}\left(x_{i}, t^{n}\right), u^{i n t, 0}\left(x_{i+1}, t^{n}\right)\right) \cdot u^{i n t, 1}\left(x_{i}, t^{n}\right) \\
& +h \partial_{2} F\left(u^{i n t, 0}\left(x_{i}, t^{n}\right), u^{i n t, 0}\left(x_{i+1}, t^{n}\right)\right) \cdot u^{i n t, 1}\left(x_{i+1}, t^{n}\right) \\
& -h \partial_{1} F\left(u^{i n t, 0}\left(x_{i-1}, t^{n}\right), u^{i n t, 0}\left(x_{i}, t^{n}\right)\right) \cdot u^{i n t, 1}\left(x_{i-1}, t^{n}\right) \\
& -h \partial_{2} F\left(u^{i n t, 0}\left(x_{i-1}, t^{n}\right), u^{i n t, 0}\left(x_{i}, t^{n}\right)\right) \cdot u^{i n t, 1}\left(x_{i}, t^{n}\right) .
\end{aligned}
$$

The term $\Delta_{F}^{0}$ will contribute to the source term in the equation on $u^{i n t, 1}$ because

$$
\Delta_{F}^{0}=h^{2} G\left(u^{i n t, 0}\left(x_{i}, t^{n}\right), \partial_{x} u^{i n t, 0}\left(x_{i}, t^{n}\right), \partial_{x x} u^{i n t, 0}\left(x_{i}, t^{n}\right)\right) .
$$

It remains to rewrite $\Delta_{F}^{1}$. Therefore, we use the consistency of the scheme $F(u, u)=f(u)$, which implies $f^{\prime}(u)=\partial_{1} F(u, u)+\partial_{2} F(u, u)$ and $f^{\prime \prime}(u)=\partial_{11} F(u, u)+2 \partial_{12} F(u, u)+\partial_{22} F(u, u)$. Developing all the derivatives of $F$ around the point $\left(u^{i n t, 0}\left(x_{i}, t^{n}\right), u^{i n t, 0}\left(x_{i}, t^{n}\right)\right)$ in $\Delta_{F}^{1}$, we get:

$$
\Delta_{F}^{1}=h^{2}\left(f^{\prime}\left(u^{i n t, 0}\left(x_{i}, t^{n}\right)\right) \cdot \partial_{x} u^{i n t, 1}\left(x_{i}, t^{n}\right)+f^{\prime \prime}\left(u^{i n t, 0}\left(x_{i}, t^{n}\right)\right) \cdot \partial_{x} u^{i n t, 0}\left(x_{i}, t^{n}\right) \cdot u^{i n t, 1}\left(x_{i}, t^{n}\right)\right)+O\left(h^{3}\right) .
$$

Finally, we find that $u^{i n t, 1}$ satisfy the linearized system of (1) around $u^{i n t, 0}$ with some source term:

$$
\partial_{t} u^{i n t, 1}+\partial_{x}\left(f^{\prime}\left(u^{i n t, 0}\right) \cdot u^{i n t, 1}\right)=S_{i n t, 1}\left(u^{i n t, 0}\right),
$$

with the initial condition $u^{i n t, 1}(x, 0)=0$, and boundary condition (23). This system is well posed, as long as $u^{i n t, 0}$ is not too large. The source term $S_{i n t, 1}$ is a function of $u^{i n t, 0}$ and its space and time derivatives until the order 2.

With the same technique, we can get the equation satisfied by all the $u^{i n t, j}$ that is:

$$
\partial_{t} u^{i n t, j}+\partial_{x}\left(f^{\prime}\left(u^{i n t, 0}\right) \cdot u^{i n t, j}\right)=S_{i n t, j}\left(u^{i n t, 0}, u^{i n t, 1}, \ldots, u^{i n t, j-1}\right)
$$

where $S_{i n t, j}$ is a function of all $u^{i n t, k}, 0 \leq k \leq j-1$ and their derivatives until the order $j+1-k$.

\subsection{About the compatibility condition (7)}

If we do not assume the compatibility of the initial condition with the numerical boundary condition (7), we have to introduce initial boundary layer profiles in the construction of $u_{\text {app }}$. Then, we search an asymptotic expansion of the form:

$$
u_{\text {app }}\left(x_{i}, t^{n}\right)=\sum_{j=0}^{N} h^{j}\left(u^{i n t, j}\left(x_{i}, t^{n}\right)+u^{b, j}\left(i, t^{n}\right)+\tilde{u}^{b, j}(i, n)\right)
$$


where the $\tilde{u}^{b, j}: \mathbb{N} \times \mathbb{N} \rightarrow \mathbb{R}^{d}$ are the initial boundary layer profiles. If the two first initial boundary layer profiles are rapidly decreasing with respect to $i$ and $n$, the results of Theorem 1.1 and Theorem 1.2 still hold.

In order to get the equations satisfied by the $\tilde{u}^{b, j}$, we develop $E_{i}^{n}$ for small $i$ and $n$ (in the initial boundary layer). It yields the equation satisfied by $\tilde{u}^{b, 0}$ :

$$
\left.\begin{array}{l}
\frac{h}{k}\left(\tilde{u}^{b, 0}(i, n+1)-\tilde{u}^{b, 0}(i, n)\right) \\
+F\left(u^{i n t, 0}(0,0)+u^{b, 0}(i, 0)+\tilde{u}^{b, 0}(i, n), u^{i n t, 0}(0,0)+u^{b, 0}(i+1,0)+\tilde{u}^{b, 0}(i+1, n)\right) \\
-F\left(u^{i n t, 0}(0,0)+u^{b, 0}(i-1,0)+\tilde{u}^{b, 0}(i-1, n), u^{i n t, 0}(0,0)+u^{b, 0}(i, 0)+\tilde{u}^{b, 0}(i, n)\right)=0 \\
\text { with } \tilde{u}^{b, 0}(i, 0)=-u^{b, 0}(i, 0) \text { and } \tilde{u}^{b, 0}(0, n)=0 .
\end{array}\right\}
$$

Note that in this equation time and space are discrete, i.e. that $\tilde{u}^{b, 0}$ is defined on $\mathbb{N} \times \mathbb{N}$. Therefore existence and uniqueness of a solution is straightforward. It remains to get decreasing properties. We will not investigate exponential decrease here and refer to [14] for related equations.

\section{Justification of The ASymptotic expansion: $L^{2}$ estimates}

In this section, we give the proof of Theorem 1.1, which is inspired by the methods of [2]. Let us consider $u_{\text {app }}$ defined by (19) where the $u^{i n t, j}$ and $u^{b, j}$ are solutions to the equations derived in the Section 2 . We want to prove that $u_{\text {app }}$ is an asymptotic expansion of the numerical solution $u_{\text {num }}$ defined by the Lax-Friedrichs scheme (12), (13), (10), (14).

Thanks to the numerical scheme, $u_{\text {num }}$ satisfies:

$\frac{h}{k}\left(u_{\text {num }}\left(x_{i}, t^{n+1}\right)-u_{\text {num }}\left(x_{i}, t^{n}\right)\right)+F\left(u_{\text {num }}\left(x_{i}, t^{n}\right), u_{\text {num }}\left(x_{i+1}, t^{n}\right)\right)-F\left(u_{\text {num }}\left(x_{i-1}, t^{n}\right), u_{\text {num }}\left(x_{i}, t^{n}\right)\right)=0$.

Furthermore, the construction of the $u^{i n t, j}$ and $u^{b, j}$ ensures that

$\frac{h}{k}\left(u_{\text {app }}\left(x_{i}, t^{n+1}\right)-u_{\text {app }}\left(x_{i}, t^{n}\right)\right)+F\left(u_{\text {app }}\left(x_{i}, t^{n}\right), u_{\text {app }}\left(x_{i+1}, t^{n}\right)\right)-F\left(u_{\text {app }}\left(x_{i-1}, t^{n}\right), u_{\text {app }}\left(x_{i}, t^{n}\right)\right)=R_{i}^{n}$

with $R_{i}^{n}=O\left(h^{N+1}\right)$ in the boundary layer and $R_{i}^{n}=O\left(h^{N+2}\right)$ outside.

We set $v=u_{\text {num }}-u_{\text {app }}$ and $v_{i}^{n}=v\left(x_{i}, t^{n}\right)$. The difference between the scheme (26) and the equation satisfied by $u_{\text {app }}(27)$ leads to:

$$
\begin{aligned}
\frac{h}{k}\left(v_{i}^{n+1}-v_{i}^{n}\right)+F\left(u_{\mathrm{num}}\left(x_{i}, t^{n}\right), u_{\mathrm{num}}\right. & \left.\left(x_{i+1}, t^{n}\right)\right)-F\left(u_{\mathrm{app}}\left(x_{i}, t^{n}\right), u_{\mathrm{app}}\left(x_{i+1}, t^{n}\right)\right) \\
& -F\left(u_{\mathrm{num}}\left(x_{i-1}, t^{n}\right), u_{\mathrm{num}}\left(x_{i}, t^{n}\right)\right)+F\left(u_{\mathrm{app}}\left(x_{i-1}, t^{n}\right), u_{\mathrm{app}}\left(x_{i}, t^{n}\right)\right)=-R_{i}^{n} .
\end{aligned}
$$

But, for the Lax-Friedrichs flux defined by (10), we have, using a Taylor's formula

$$
\begin{aligned}
& F\left(u_{\mathrm{num}}\left(x_{i}, t^{n}\right), u_{\mathrm{num}}\left(x_{i+1}, t^{n}\right)\right)-F\left(u_{\mathrm{app}}\left(x_{i}, t^{n}\right), u_{\mathrm{app}}\left(x_{i+1}, t^{n}\right)\right)= \\
& \frac{f^{\prime}\left(u_{\mathrm{app}}\left(x_{i}, t^{n}\right)\right) v_{i}^{n}+f^{\prime}\left(u_{\mathrm{app}}\left(x_{i+1}, t^{n}\right)\right) v_{i+1}^{n}}{2}-\frac{\lambda}{2}\left(v_{i+1}^{n}-v_{i}^{n}\right)+C_{i}^{n} v_{i}^{n} v_{i}^{n}+C_{i+1}^{n} v_{i+1}^{n} v_{i+1}^{n},
\end{aligned}
$$

where $\left|C_{i}^{n}\right| \leq C$ because the second derivatives of $f$ are uniformly bounded.

Let us introduce

$$
A_{i}^{n}=f^{\prime}\left(u_{\mathrm{app}}\left(x_{i}, t^{n}\right)\right), \quad \mathcal{A}_{i, n}^{+}=\frac{A_{i}^{n}+\lambda I}{2}, \quad \mathcal{A}_{i, n}^{-}=\frac{A_{i}^{n}-\lambda I}{2}
$$


The $v_{i}^{n}$ are solutions of a kind of "linearized" scheme with error terms

$$
\frac{h}{k}\left(v_{i}^{n+1}-v_{i}^{n}\right)+\mathcal{A}_{i, n}^{+} v_{i}^{n}+\mathcal{A}_{i+1, n}^{-} v_{i+1}^{n}-\mathcal{A}_{i-1, n}^{+} v_{i-1}^{n}-\mathcal{A}_{i, n}^{-} v_{i}^{n}=-T_{i}^{n}-R_{i}^{n}
$$

where $\left|T_{i}^{n}\right| \leq C\left(\left|v_{i}^{n}\right|^{2}+\left|v_{i+1}^{n}\right|^{2}+\left|v_{i-1}^{n}\right|^{2}\right)$ and with the initial condition $v_{i}^{0}=0$ for all $i \in \mathbb{N}$.

Using the hypothesis of symmetrizability of the system (9), we also introduce $\mathcal{S}_{i}^{n}=\mathcal{S}\left(u_{\text {app }}\left(x_{i}, t^{n}\right)\right)$. The matrix $\mathcal{S}_{i}^{n}$ is symmetric nonnegative definite, $\mathcal{S}_{i}^{n} \gg I$ and $\mathcal{S}_{i}^{n} A_{i}^{n}$ is symmetric. Moreover, $\mathcal{S}_{i}^{n}$ is uniformly bounded. As $\mathcal{S}_{i}^{n}$ is symmetric nonnegative definite, there exists $S_{i}^{n}$ symmetric nonnegative definite such that $\left(S_{i}^{n}\right)^{2}=\mathcal{S}_{i}^{n}$ and, for all $i \in \mathbb{N}$, for all $n \in \mathbb{N}$, we have the following norm equivalence:

$$
\forall v \in \mathbb{R}^{d},|v|^{2} \leq\left|S_{i}^{n} v\right|^{2} \leq C|v|^{2} .
$$

Multiplying (29) by $S_{i}^{n}$, we get:

$$
S_{i}^{n} v_{i}^{n+1}=S_{i}^{n} v_{i}^{n}-\frac{k}{h}\left(F_{1}+F_{2}+F_{3}\right)
$$

with

$$
\begin{aligned}
& F_{1}=S_{i}^{n} \mathcal{A}_{i, n}^{+}\left(v_{i}^{n}-v_{i-1}^{n}\right)+S_{i}^{n} \mathcal{A}_{i, n}^{-}\left(v_{i+1}^{n}-v_{i}^{n}\right), \\
& F_{2}=S_{i}^{n}\left(\mathcal{A}_{i+1, n}^{-}-\mathcal{A}_{i, n}^{-}\right) v_{i+1}^{n}+S_{i}^{n}\left(\mathcal{A}_{i, n}^{+}-\mathcal{A}_{i-1, n}^{+}\right) v_{i-1}^{n}, \\
& F_{3}=S_{i}^{n} T_{i}^{n}+S_{i}^{n} R_{i}^{n} .
\end{aligned}
$$

Therefore,

and

$$
\left|S_{i}^{n} v_{i}^{n+1}\right|^{2}=\left|S_{i}^{n} v_{i}^{n}\right|^{2}-2 \frac{k}{h} S_{i}^{n} v_{i}^{n} \cdot\left(F_{1}+F_{2}+F_{3}\right)+\frac{k^{2}}{h^{2}}\left|F_{1}+F_{2}+F_{3}\right|^{2}
$$

$$
\left|S_{i}^{n} v_{i}^{n+1}\right|^{2} \leq\left|S_{i}^{n} v_{i}^{n}\right|^{2}-2 \frac{k}{h} S_{i}^{n} v_{i}^{n} \cdot\left(F_{1}+F_{2}+F_{3}\right)+\frac{3 k^{2}}{h^{2}}\left(\left|F_{1}\right|^{2}+\left|F_{2}\right|^{2}+\left|F_{3}\right|^{2}\right) .
$$

We now bound all the terms of the right hand side of (32).

1. Terms containing $F_{1}$

First, we rewrite

$$
2 S_{i}^{n} v_{i}^{n} \cdot F_{1}=2 v_{i}^{n} \cdot \mathcal{S}_{i}^{n} \mathcal{A}_{i, n}^{+}\left(v_{i}^{n}-v_{i-1}^{n}\right)+2 v_{i}^{n} \cdot \mathcal{S}_{i}^{n} \mathcal{A}_{i, n}^{-}\left(v_{i+1}^{n}-v_{i}^{n}\right) .
$$

But, thanks to $(9), \mathcal{S}_{i}^{n} \mathcal{A}_{i, n}^{+}$and $\mathcal{S}_{i}^{n} \mathcal{A}_{i, n}^{-}$are symmetric matrices and for a symmetric matrix $M$, we have

$$
2 a \cdot M(a-b)=a \cdot M a-b \cdot M b+(a-b) \cdot M(a-b), \quad \forall a, b \in \mathbb{R}^{d} .
$$

Thus,

$$
\begin{aligned}
2 S_{i}^{n} v_{i}^{n} \cdot F_{1}=v_{i}^{n} \cdot \mathcal{S}_{i}^{n} \mathcal{A}_{i, n}^{+} v_{i}^{n}-v_{i-1}^{n} \cdot \mathcal{S}_{i}^{n} \mathcal{A}_{i, n}^{+} v_{i-1}^{n}+\left(v_{i}^{n}-v_{i-1}^{n}\right) \cdot \mathcal{S}_{i}^{n} \mathcal{A}_{i, n}^{+}\left(v_{i}^{n}-v_{i-1}^{n}\right) & \\
& +v_{i+1}^{n} \cdot \mathcal{S}_{i}^{n} \mathcal{A}_{i, n}^{-} v_{i+1}^{n}-v_{i}^{n} \mathcal{S}_{i}^{n} \mathcal{A}_{i, n}^{-} v_{i}^{n}-\left(v_{i}^{n}-v_{i+1}^{n}\right) \cdot \mathcal{S}_{i}^{n} \mathcal{A}_{i, n}^{-}\left(v_{i}^{n}-v_{i+1}^{n}\right) .
\end{aligned}
$$

Moreover,

$$
\left|F_{1}\right|^{2} \leq 2\left(\left|S_{i}^{n} \mathcal{A}_{i, n}^{+}\left(v_{i}^{n}-v_{i-1}^{n}\right)\right|^{2}+\left|S_{i}^{n} \mathcal{A}_{i, n}^{-}\left(v_{i+1}^{n}-v_{i}^{n}\right)\right|^{2}\right)
$$


2. Terms containing $F_{2}$

By definition, we have $2\left(\mathcal{A}_{i+1, n}^{-}-\mathcal{A}_{i, n}^{-}\right)=A_{i+1}^{n}-A_{i}^{n}=f^{\prime}\left(u_{\text {app }}\left(x_{i+1}, t^{n}\right)\right)-f^{\prime}\left(u_{\text {app }}\left(x_{i}, t^{n}\right)\right)$ and $2\left(\mathcal{A}_{i, n}^{+}-\right.$ $\left.\mathcal{A}_{i-1, n}^{+}\right)=A_{i}^{n}-A_{i-1}^{n}=f^{\prime}\left(u_{\text {app }}\left(x_{i}, t^{n}\right)\right)-f^{\prime}\left(u_{\text {app }}\left(x_{i-1}, t^{n}\right)\right)$. The regularity of $u^{i n t, 0}$ and $u^{b, 0}$ (we assume here that the decrease of $u^{b, 0}$ is exponential) implies

$$
\begin{aligned}
\left|\mathcal{A}_{i+1, n}^{-}-\mathcal{A}_{i, n}^{-}\right| & \leq C_{i n t} h+C_{b} \mathrm{e}^{-\mu i} \\
\left|\mathcal{A}_{i, n}^{+}-\mathcal{A}_{i-1, n}^{+}\right| & \leq C_{i n t} h+C_{b} \mathrm{e}^{-\mu i} .
\end{aligned}
$$

An important point to note is that $C_{b}$ depends on $\left|u^{i n t, 0}(0, t)\right|$ and goes to 0 as $\left|u^{i n t, 0}(0, t)\right|$ goes to 0 . We have

$$
\begin{aligned}
2\left|S_{i}^{n} v_{i}^{n} \cdot F_{2}\right| & \leq C\left(C_{i n t} h+C_{b} \mathrm{e}^{-\mu i}\right)\left(\left|v_{i+1}^{n}\right|^{2}+\left|v_{i}^{n}\right|^{2}+\left|v_{i-1}^{n}\right|^{2}\right), \\
\left|F_{2}\right|^{2} & \leq C\left(C_{i n t} h+C_{b} \mathrm{e}^{-\mu i}\right)\left(\left|v_{i+1}^{n}\right|^{2}+\left|v_{i-1}^{n}\right|^{2}\right) .
\end{aligned}
$$

3. Terms containing $F_{3}$

For $F_{3}$, we have the following bounds:

$$
\begin{aligned}
2\left|S_{i}^{n} v_{i}^{n} \cdot F_{3}\right| & \leq C\left(\left|v_{i}^{n}\right|^{3}+\left|v_{i+1}^{n}\right|^{3}+\left|v_{i-1}^{n}\right|^{3}+\left|v_{i}^{n}\right|\left|R_{i}^{n}\right|\right) \\
\left|F_{3}\right|^{2} & \leq C\left(\left|v_{i}^{n}\right|^{4}+\left|v_{i+1}^{n}\right|^{4}+\left|v_{i-1}^{n}\right|^{4}+\left|R_{i}^{n}\right|^{2}\right) .
\end{aligned}
$$

Introducing all these bounds in (32), we get:

$$
\begin{aligned}
\left|S_{i}^{n} v_{i}^{n+1}\right|^{2} \leq\left|S_{i}^{n} v_{i}^{n}\right|^{2} & -\frac{k}{h}\left(v_{i+1}^{n} \cdot \mathcal{S}_{i}^{n} \mathcal{A}_{i, n}^{-} v_{i+1}^{n}-v_{i}^{n} \cdot \mathcal{S}_{i}^{n} \mathcal{A}_{i, n}^{-} v_{i}^{n}\right) \\
& -\frac{k}{h}\left(v_{i}^{n} \cdot \mathcal{S}_{i}^{n} \mathcal{A}_{i, n}^{+} v_{i}^{n}-v_{i-1}^{n} \cdot \mathcal{S}_{i}^{n} \mathcal{A}_{i, n}^{+} v_{i-1}^{n}\right) \\
& -\frac{k}{h}\left(v_{i}^{n}-v_{i-1}^{n}\right) \cdot \mathcal{S}_{i}^{n} \mathcal{A}_{i, n}^{+}\left(v_{i}^{n}-v_{i-1}^{n}\right)+\frac{6 k^{2}}{h^{2}}\left|S_{i}^{n} \mathcal{A}_{i, n}^{+}\left(v_{i}^{n}-v_{i-1}^{n}\right)\right|^{2} \\
& +\frac{k}{h}\left(v_{i}^{n}-v_{i+1}^{n}\right) \cdot \mathcal{S}_{i}^{n} \mathcal{A}_{i, n}^{-}\left(v_{i}^{n}-v_{i+1}^{n}\right)+\frac{6 k^{2}}{h^{2}}\left|S_{i}^{n} \mathcal{A}_{i, n}^{-}\left(v_{i}^{n}-v_{i+1}^{n}\right)\right|^{2} \\
& +C \frac{k}{h}\left(C_{i n t} h+C_{b} \mathrm{e}^{-\mu i}\right)\left(\left|v_{i+1}^{n}\right|^{2}+\left|v_{i}^{n}\right|^{2}+\left|v_{i-1}^{n}\right|^{2}\right) \\
& +C \frac{k}{h}\left(\left|v_{i}^{n}\right|^{3}+\left|v_{i+1}^{n}\right|^{3}+\left|v_{i-1}^{n}\right|^{3}+\left|v_{i}^{n}\right|^{4}+\left|v_{i+1}^{n}\right|^{4}+\left|v_{i-1}^{n}\right|^{4}\right) \\
& +C \frac{k}{h}\left(\left|v_{i}^{n}\right|\left|R_{i}^{n}\right|+\left|R_{i}^{n}\right|^{2}\right) .
\end{aligned}
$$

But, for a large $\lambda, \mathcal{S}_{i}^{n} \mathcal{A}_{i, n}^{+}$is symmetric nonnegative definite with eigenvalues greater than $\gamma$ (depending on $\lambda$ ) and $\mathcal{S}_{i}^{n} \mathcal{A}_{i, n}^{-}$is symmetric non positive definite with eigenvalues smaller than $-\gamma$. Moreover, $\left|S_{i}^{n} \mathcal{A}_{i, n}^{+}\right|$and $\left|S_{i}^{n} \mathcal{A}_{i, n}^{-}\right|$are bounded by $M$ (depending on $\lambda$ ).

Therefore, under the CFL condition

$$
\left.\frac{k}{h} \leq \gamma \frac{1-\xi}{6 M^{2}}, \xi \in\right] 0,1[
$$

we have

and

$$
-\frac{k}{h}\left(v_{i}^{n}-v_{i-1}^{n}\right) \cdot \mathcal{S}_{i}^{n} \mathcal{A}_{i, n}^{+}\left(v_{i}^{n}-v_{i-1}^{n}\right)+\frac{6 k^{2}}{h^{2}}\left|S_{i}^{n} \mathcal{A}_{i, n}^{+}\left(v_{i}^{n}-v_{i-1}^{n}\right)\right|^{2} \leq-\gamma \xi \frac{k}{h}\left|v_{i}^{n}-v_{i-1}^{n}\right|^{2}
$$

$$
\frac{k}{h}\left(v_{i}^{n}-v_{i+1}^{n}\right) \cdot \mathcal{S}_{i}^{n} \mathcal{A}_{i, n}^{-}\left(v_{i}^{n}-v_{i+1}^{n}\right)+\frac{6 k^{2}}{h^{2}}\left|S_{i}^{n} \mathcal{A}_{i, n}^{-}\left(v_{i}^{n}-v_{i+1}^{n}\right)\right|^{2} \leq-\gamma \xi \frac{k}{h}\left|v_{i}^{n}-v_{i+1}^{n}\right|^{2} .
$$


Thus, we get

$$
\begin{aligned}
\sum_{i \geq 1}\left|S_{i}^{n} v_{i}^{n+1}\right|^{2} \leq \sum_{i \geq 1}\left|S_{i}^{n} v_{i}^{n}\right|^{2} & -\frac{k}{h} \sum_{i \geq 1}\left(v_{i}^{n} \cdot\left(\mathcal{S}_{i-1}^{n} \mathcal{A}_{i-1, n}^{-}-\mathcal{S}_{i}^{n} \mathcal{A}_{i, n}^{-}\right) v_{i}^{n}\right) \\
& -\frac{k}{h} \sum_{i \geq 1}\left(v_{i}^{n} \cdot\left(\mathcal{S}_{i+1}^{n} \mathcal{A}_{i+1, n}^{+}-\mathcal{S}_{i}^{n} \mathcal{A}_{i, n}^{+}\right) v_{i}^{n}\right) \\
& -\gamma \frac{\xi k}{h} \sum_{i \geq 1}\left|v_{i}^{n}-v_{i-1}^{n}\right|^{2}-\gamma \frac{\xi k}{h} \sum_{i \geq 1}\left|v_{i}^{n}-v_{i+1}^{n}\right|^{2} \\
& +C \frac{k}{h}\left(C_{i n t} h \sum_{i \geq 1}\left|v_{i}^{n}\right|^{2}+C \sum_{i \geq 1} \mathrm{e}^{-\mu i}\left|v_{i}^{n}\right|^{2}\right) \\
& +C \frac{k}{h} \sum_{i \geq 1}\left(\left|v_{i}^{n}\right|^{3}+\left|v_{i}^{n}\right|^{4}\right)+C \frac{k}{h}\left(\sum_{i \geq 1}\left|v_{i}^{n}\right|\left|R_{i}^{n}\right|+\sum_{i \geq 1}\left|R_{i}^{n}\right|^{2}\right)
\end{aligned}
$$

and further:

$$
\begin{aligned}
\sum_{i \geq 1}\left|S_{i}^{n} v_{i}^{n+1}\right|^{2} \leq \sum_{i \geq 1}\left|S_{i}^{n} v_{i}^{n}\right|^{2}\left(1+C \frac{k}{h} h\right)+C_{b} \frac{k}{h} \sum_{i \geq 1} \mathrm{e}^{-\mu i}\left|v_{i}^{n}\right|^{2}-C_{d} \frac{k}{h} \sum_{i \geq 1}\left|v_{i}^{n}-v_{i-1}^{n}\right|^{2} & \\
& +C \frac{k}{h} \sum_{i \geq 1}\left(\left|v_{i}^{n}\right|^{3}+\left|v_{i}^{n}\right|^{4}\right)+C \frac{k}{h}\left(\sum_{i \geq 1}\left|v_{i}^{n}\right|\left|R_{i}^{n}\right|+\sum_{i \geq 1}\left|R_{i}^{n}\right|^{2}\right)
\end{aligned}
$$

But, we can control the term $C_{b} \sum_{i \geq 1} \mathrm{e}^{-\mu i}\left|v_{i}^{n}\right|^{2}$ by $C_{d} \sum_{i \geq 1}\left|v_{i}^{n}-v_{i-1}^{n}\right|^{2}$ so that their difference stays nonpositive. Indeed, as $v_{0}^{n}=0, v_{i}^{n}=\sum_{j \leq i}\left(v_{j}^{n}-v_{j-1}^{n}\right)$ and

$$
\left|v_{i}^{n}\right|^{2} \leq i \sum_{j \leq i}\left|v_{j}^{n}-v_{j-1}^{n}\right|^{2} \leq i \sum_{j \geq 1}\left|v_{j}^{n}-v_{j-1}^{n}\right|^{2}
$$

hence

$$
\sum_{i \geq 1} \mathrm{e}^{-\mu i}\left|v_{i}^{n}\right|^{2} \leq\left(\sum_{i \geq 1} i \mathrm{e}^{-\mu i}\right) \sum_{i \geq 1}\left|v_{i}^{n}-v_{i-1}^{n}\right|^{2}
$$

Thus, if

$$
C_{b} \leq \frac{C_{d}}{\sum_{i \geq 1} i \mathrm{e}^{-\mu i}}
$$

we deduce from (34)

$$
\sum_{i \geq 1}\left|S_{i}^{n} v_{i}^{n+1}\right|^{2} \leq \sum_{i \geq 1}\left|S_{i}^{n} v_{i}^{n}\right|^{2}(1+C k)+C \frac{k}{h} \sum_{i \geq 1}\left(\left|v_{i}^{n}\right|^{3}+\left|v_{i}^{n}\right|^{4}\right)+C \frac{k}{h}\left(\sum_{i \geq 1}\left|v_{i}^{n}\right|\left|R_{i}^{n}\right|+\sum_{i \geq 1}\left|R_{i}^{n}\right|^{2}\right)
$$

Note again that $(35)$ is satisfied if $\sup _{t}\left|u^{i n t, 0}(0, t)\right|$ is small enough.

Now, we want to replace $\sum_{i \geq 1}\left|S_{i}^{n} v_{i}^{n+1}\right|^{2}$ in the last inequality by $\sum_{i \geq 1}\left|S_{i}^{n+1} v_{i}^{n+1}\right|^{2}$. But,

$$
S_{i}^{n+1} v_{i}^{n+1}=\left(S_{i}^{n+1}-S_{i}^{n}\right) v_{i}^{n+1}+S_{i}^{n} v_{i}^{n+1}
$$


and for all $n \in \mathbb{N},\left|S_{i}^{n+1}-S_{i}^{n}\right| \leq C k$. Thus, thanks to (30), we get

$$
\left|S_{i}^{n+1} v_{i}^{n+1}\right|^{2} \leq\left|S_{i}^{n} v_{i}^{n+1}\right|^{2}+C k\left|S_{i}^{n+1} v_{i}^{n+1}\right|^{2}
$$

Therefore,

$$
\sum_{i \geq 1}\left|S_{i}^{n+1} v_{i}^{n+1}\right|^{2} \leq \frac{1}{1-C k} \sum_{i \geq 1}\left|S_{i}^{n} v_{i}^{n+1}\right|^{2} \leq\left(1+C^{\prime} k\right) \sum_{i \geq 1}\left|S_{i}^{n} v_{i}^{n+1}\right|^{2}
$$

and for all $n \in \mathbb{N}$, we have

$$
\sum_{i \geq 1}\left|S_{i}^{n+1} v_{i}^{n+1}\right|^{2} \leq(1+C k) \sum_{i \geq 1}\left|S_{i}^{n} v_{i}^{n}\right|^{2}+C \frac{k}{h} \sum_{i \geq 1}\left(\left|v_{i}^{n}\right|^{3}+\left|v_{i}^{n}\right|^{4}\right)+C \frac{k}{h}\left(\sum_{i \geq 1}\left|v_{i}^{n}\right|\left|R_{i}^{n}\right|+\sum_{i \geq 1}\left|R_{i}^{n}\right|^{2}\right)
$$

Let introduce $N_{h}=\sup \left\{n ; \sum_{i \geq 1}\left|S_{i}^{n} v_{i}^{n}\right|^{2} \leq h^{2}\right\}$ and $T_{h}=N_{h} k$. For all $n \leq N_{h}$, we have

$$
\begin{aligned}
\left|v_{i}^{n}\right| & \leq\left|S_{i}^{n} v_{i}^{n}\right| \leq h \\
\sum_{i \geq 1}\left|v_{i}^{n}\right|^{3} & \leq h \sum_{i \geq 1}\left|S_{i}^{n} v_{i}^{n}\right|^{2} \\
\sum_{i \geq 1}\left|v_{i}^{n}\right|^{4} & \leq h \sum_{i \geq 1}\left|S_{i}^{n} v_{i}^{n}\right|^{2} \\
\sum_{i \geq 1}\left|v_{i}^{n}\right|\left|R_{i}^{n}\right| & \leq h \sum_{i \geq 1}\left|S_{i}^{n} v_{i}^{n}\right|^{2}+\sum_{i \geq 1} \frac{\left|R_{i}^{n}\right|^{2}}{h} .
\end{aligned}
$$

But, we proved that $\left|R_{i}^{n}\right| \leq C h^{N+1}$ in the boundary layer and $\left|R_{i}^{n}\right| \leq C h^{N+2}$ outside.

Therefore, if $u_{0}$ has a compact support,

$$
\sum_{i \geq 1}\left|R_{i}^{n}\right|^{2} \leq C h^{2 N+2}
$$

From (36), we deduce that, for all $n \in \mathbb{N}$,

$$
\sum_{i \geq 1}\left|S_{i}^{n+1} v_{i}^{n+1}\right|^{2} \leq(1+C k) \sum_{i \geq 1}\left|S_{i}^{n} v_{i}^{n}\right|^{2}+C k h^{2 N}
$$

We can then apply a discrete Gronwall lemma. As $v_{i}^{0}=0$ for all $i \in \mathbb{N}$, we finally get

$$
\sum_{i \geq 1}\left|S_{i}^{n} v_{i}^{n}\right|^{2} \leq C(1+C k)^{n} n k h^{2 N} \leq C(1+C k)^{\frac{T_{h}}{k}} T_{h} h^{2 N}, \forall n \leq N_{h}
$$

We deduce that, if $u_{\text {app }}$ is computed until order $N \geq 2, T_{h} \geq T$ where $T$ is the existence time for a regular solution to the hyperbolic problem (1). It also proves in this case, that, on $[0, T]$,

$$
\left\|u_{\text {app }}\left(., t^{n}\right)-u_{\text {num }}\left(., t^{n}\right)\right\|_{L^{2}} \leq h .
$$

\section{THE SCALAR CASE}

Let us now prove Theorem 1.2. This theorem holds for every scheme satisfying the hypotheses of monotonicity and consistency (11), without any smallness condition. 
The first step is to construct an approximate solution $u_{\text {app }}$ of the form (19) for $N \geq 2$ : it is done in Section 2 . The second step is to make energy estimates on $v_{i}^{n}=u_{\text {num }}\left(x_{i}, t^{n}\right)-u_{\text {app }}\left(x_{i}, t^{n}\right)$. The main ingredient is a trick of Goodman [7] and Liu [11]: we integrate the equation in $x$ and work on

$$
\partial_{t} U+f\left(\partial_{x} U\right)=0
$$

where $\partial_{x} U=u$, instead of working on (1). This change in the formulation makes a crucial change in the energy $L^{2}$ estimate (see [7]), by changing a sign.

At the discrete level, we introduce $U_{i}^{n}=-\sum_{j \geq i} u_{j}^{n}$, and similarly $V_{i}^{n}$ and $U_{i}^{\text {app, }, n}$. The scheme rewrites

$$
\frac{h}{k}\left(U_{i}^{n+1}-U_{i}^{n}\right)+F\left(U_{i}^{n}-U_{i-1}^{n}, U_{i+1}^{n}-U_{i}^{n}\right)=0
$$

Furthermore, the construction of $u_{\text {app }}$ ensures

$$
\frac{h}{k}\left(U_{i}^{\mathrm{app}, n+1}-U_{i}^{\mathrm{app}, n}\right)+F\left(U_{i}^{\mathrm{app}, n}-U_{i-1}^{\mathrm{app}, n}, U_{i+1}^{\mathrm{app}, n}-U_{i}^{\mathrm{app}, n}\right)=R_{i}^{n}
$$

where $R_{i}^{n}=O\left(h^{N+1}\right)$ in the boundary and initial boundary layers and $R_{i}^{n}=O\left(h^{N+2}\right)$ outside. Therefore,

$$
\begin{aligned}
\frac{h}{k}\left(V_{i}^{n+1}-V_{i}^{n}\right)+\partial_{1} F\left(U_{i}^{\mathrm{app}, n}-U_{i-1}^{\mathrm{app}, n}\right. & \left., U_{i+1}^{\mathrm{app}, n}-U_{i}^{\mathrm{app}, n}\right)\left(V_{i}^{n}-V_{i-1}^{n}\right) \\
& +\partial_{2} F\left(U_{i}^{\mathrm{app}, n}-U_{i-1}^{\mathrm{app}, n}, U_{i+1}^{\mathrm{app}, n}-U_{i}^{\mathrm{app}, n}\right)\left(V_{i+1}^{n}-V_{i}^{n}\right)=T_{i}^{n}+R_{i}^{n}
\end{aligned}
$$

where, using a Taylor formula,

$$
\left|T_{i}^{n}\right| \leq C\left(\left|V_{i}^{n}\right|^{2}+\left|V_{i-1}^{n}\right|^{2}+\left|V_{i+1}^{n}\right|^{2}\right)
$$

Using the monotonicity of the scheme we get

$$
\left\|V^{n+1}\right\|_{L^{\infty}} \leq\left\|V^{n}\right\|_{L^{\infty}}+C \frac{k}{h}\left\|V^{n}\right\|_{L^{\infty}}^{2}+C k h^{N}
$$

with $V(0)=0$ and the proof can be ended as in the previous section, using a discrete Gronwall type lemma.

\section{Numerical COUNTEREXAMPLES}

Numerical examples of stable boundary layers can be found in [6] for the Godunov and Lax Friedrichs schemes. In this section we will give counterexamples, that is examples of unstable boundary layers.

The first one is inspired by the unstable layer described in [8], but is not in conservative form. Let us consider the following system

$$
\begin{gathered}
\partial_{t} u+\partial_{x} u-u \partial_{x} v+\partial_{x} \frac{u^{2}}{2}=0, \\
\partial_{t} v-\partial_{x} v=0,
\end{gathered}
$$

and let try to compute the solutions using a Lax Friedrichs type scheme, starting from a small $u$ (of order $10^{-3}$ to fix the ideas) and $v=\mu \geq 0$. For small $\mu$, Theorem 1.1 can be applied and $v$ has a boundary layer type behavior (with an exponentially decreasing boundary layer) and $u$ remains small, of order $10^{-3}$. However if $\mu$ is large enough, $v$ has always a boundary layer type behavior, but $u=0$ is unstable. Therefore $u$ begins to grow in the vicinity of 0 . This comes from the term $u \partial_{x} v$ since $\partial_{x} v$ is very large in the layer, and $u \partial_{x} v$ acts as 


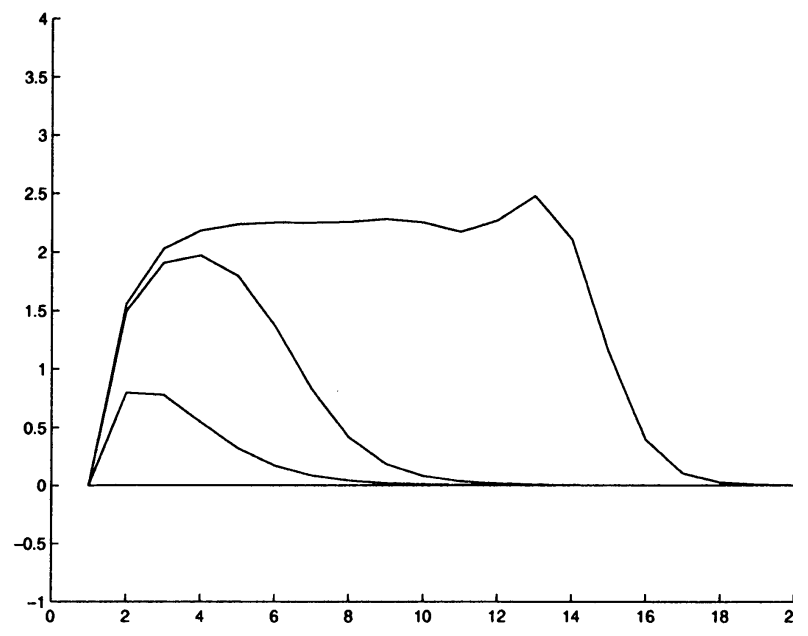

Figure 1. Nonconservative case: $u$ and $v$ at different times.

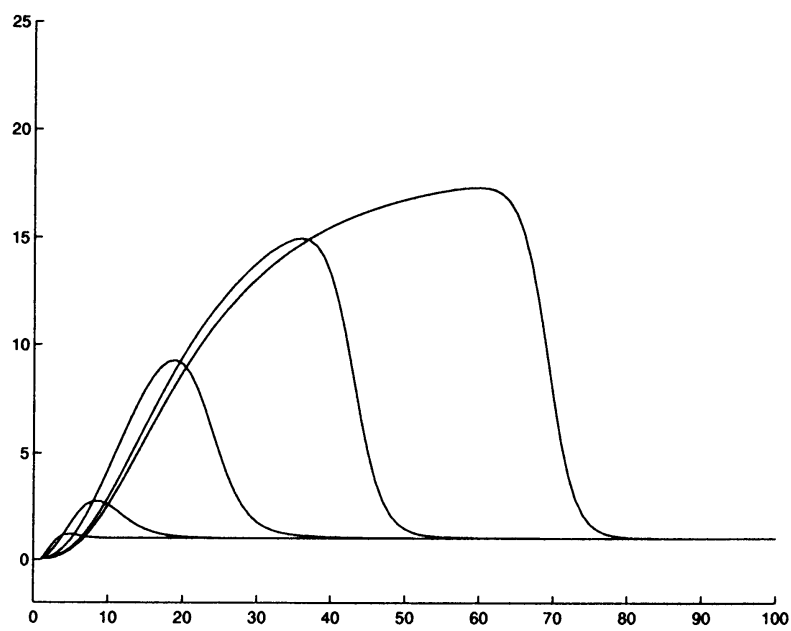

Figure 2. Conservative case: $u$ and $v$ at different times.

an important amplification term. The $\partial_{x} u$ term causes the instability to leave $x=0$ and soon a shock appears, of very high strength, which propagates in the interior. The shock simply comes from a nonlinear saturation effect of the Hopf term $\partial_{x} u^{2} / 2$. Figure 1 shows the behavior of $v$ and $u$ at different times in the boundary layer (initial data, during the growth in the boundary layer, formation of the shock, after a while the shock is inside the domain).

Therefore if try to compute the obvious solution $u=0$ and $v=\mu$ for $\mu$ large enough using a Lax Friedrichs type scheme, we go into big troubles, since instead of $u=0$ we get a big shock which enters the domain!

The second example is conservative. Let us consider the system

$$
\begin{gathered}
\partial_{t} u+\partial_{x} u-20 \partial_{x}(u(v-1 / 2))+\partial_{x} \frac{u^{2}}{2}=0, \\
\partial_{t} v-\partial_{x} v=0,
\end{gathered}
$$


with initial data $v=1$ and $u=\mu$. Then for large $\mu$ we get an instability similar to the previous one (see Fig. 2 for a zoom on the boundary layer).

Acknowledgements. The authors would like to thank Denis Serre for bringing this problem to their attention and for interesting discussions.

\section{REFERENCES}

[1] C. Bardos, A.-Y. Leroux and J.-C. Nédélec, First order quasilinear equations with boundary conditions. Partial Differential Equations 4 (1979) 1017-1034.

[2] Y. Coudière, J.-P. Vila and P. Villedieu, Convergence of a finite-volume time-explicit scheme for symmetric linear hyperbolic systems on bounded domains. C. R. Acad. Sci. Paris, Sér. I Math. 331 (2000) 95-100.

[3] F. Dubois and P. LeFloch, Boundary conditions for nonlinear hyperbolic systems of conservation laws. J. Differential Equations 71 (1988) 93-122.

[4] M. Gisclon, Étude des conditions aux limites pour un système strictement hyperbolique, via l'approximation parabolique. J. Math. Pures Appl. 75 (1996) 485-508.

[5] M. Gisclon and D. Serre, Étude des conditions aux limites pour un système strictement hyberbolique via l'approximation parabolique. C. R. Acad. Sci. Paris Sér. I Math. 319 (1994) 377-382.

[6] M. Gisclon and D. Serre, Conditions aux limites pour un système strictement hyperbolique fournies par le schéma de Godunov. RAIRO-Modél. Math. Anal. Numér. 31 (1997) 359-380.

[7] J. Goodman, Nonlinear asymptotic stability of viscous shock profiles for conservation laws. Arch. Rational Mech. Anal. 95 (1986) 325-344.

[8] E. Grenier and O. Guès, Boundary layers for viscous perturbations of noncharacteristic quasilinear hyperbolic problems. J. Differential Equations 143 (1998) 110-146.

[9] K.T. Joseph and P.G. LeFloch, Boundary layers in weak solutions of hyperbolic conservation laws. Arch. Ration. Mech. Anal. 147 (1999) 47-88.

[10] T.T. Li and W.C. Yu, Boundary value problems for quasilinear hyperbolic systems. Math. series V. Duke Univ., Durham (1985).

[11] T.P. Liu, Nonlinear stability of shock waves for viscous conservation laws. Mem. Amer. Math. Soc. 56 (1985) 108 p.

[12] J.B. Rauch and F.J. Massey, III, Differentiability of solutions to hyperbolic initial boundary value problems. Trans. Amer. Math. Soc. 189 (1974) 303-318.

[13] D. Serre, Sur la stabilité des couches limites de viscosité, preprint.

[14] M. Shub, A. Fathi and R. Langevin, Global stability of dynamical systems. Springer-Verlag, New-York, Berlin, 1987.

To access this journal online:

www.edpsciences.org 